\title{
Assignment Rules of a Single Indivisible Object under the Full Preference Domain
}

\author{
C. Gizem Korpeoglu \\ University College London, London, UK WC1E 6BT \\ c.korpeoglu@ucl.ac.uk
}

November 17, 2015

\begin{abstract}
We consider the allocation problem of a single indivisible object to one of several agents under the full preference domain when monetary transfers are not allowed. Our central requirement is strategy-proofness. The additional properties we seek are Pareto optimality, non-dictatorship, and non-bossiness. We provide characterizations of strategy-proof rules that satisfy two out of three additional properties: Pareto optimality and non-bossiness; non-dictatorship and non-bossiness; and Pareto optimality and non-dictatorship. As a consequence of these characterizations, we show that a strategy-proof rule cannot satisfy these three additional properties simultaneously.
\end{abstract}

Keywords: Indivisible Object; Strategy-proofness; Pareto Optimality; Full Preference Domain.

JEL codes: C78, D71, D78.

\section{Introduction}

We consider the following problem. A central agency is to allocate a single indivisible object (a good or a bad) among a set of agents where monetary transfers are not possible. Some examples of such objects are an organ to patients, a public school seat to students, and a task to employees. The single indivisible object is owned by the central agency, and we study its allocation problem under the full preference domain. In particular, an agent may prefer to receive the object or prefer not to receive it or be indifferent between two. In real life, agents are often indifferent when they do not know the quality or the features of an object. In such situations, it may be costly to restrict the full preference domain to the strict one by treating indifferent agents as if they strictly prefer to receive the object or strictly prefer not to. For example, an agent is indifferent to the object, he is considered as 'preferring' it under the strict preference domain, and he receives the object. When there are other agents who actually prefer to obtain the object, assigning it to an indifferent agent will result in an efficiency loss. Similarly, if the indifferent agent is considered as 'not 
preferring' the object under the strict preference domain, an efficient rule requires that the object is not assigned to this agent. This requirement introduces an artificial constraint for an efficient rule, which unnecessarily restricts the set of efficient rules when there are other agents who actually prefer not to receive the object. Erdil and Ergin (2008) establish this result by showing that random tie-breaking in school choice districts in the United States adversely affects the welfare of students by introducing artificial constraints.

In this paper, our main requirement is strategy-proofness, which means that no agent can gain from reporting false preference. This strategic robustness property has been widely used to guarantee that agents reveal their true preferences. ${ }^{1}$ In addition to strategy-proofness, we seek the following properties: Pareto optimality, non-dictatorship, and non-bossiness. Pareto optimality is a standard efficiency notion used in almost all economic applications, non-dictatorship and nonbossiness are fairness properties. We define the three additional criteria as follows. A rule is Pareto optimal if there is no other rule that provides weakly better outcomes for all agents and strictly better outcomes for some agents. A rule is non-dictatorial if there is no agent who receives the object whenever he prefers to do so and does not receive it whenever he prefers not to. A rule is nonbossy if there is no agent who can change the outcome of another agent without changing his own outcome.

Our analysis yields the following results. We first show that Pareto optimality implies strategyproofness (Proposition 1). We prove that a strategy-proof and non-bossy rule has a ranking function (Proposition 2). We then characterize strategy-proof rules as follows. A Pareto optimal and non-bossy rule is dictatorial (Proposition 3), a non-dictatorial and non-bossy rule is suboptimal (Proposition 4), and a Pareto optimal and non-dictatorial rule is bossy (Proposition 5). Hence, we show that a strategy-proof rule cannot satisfy Pareto optimality, non-dictatorship and non-bossiness simultaneously (Proposition 6).

\subsection{Related Literature}

This paper belongs to the literature on the allocation problem of an indivisible object. This problem goes back to Glazer and Ma (1989), who consider allocating a prize to the agent who values it most without monetary transfers, and construct multistage mechanisms with a unique (subgame perfect) equilibrium. Perry and Reny (1999) and Olszewski (2003) generalize the results of Glazer and Ma (1989) by reducing informational restrictions. In a recent study, Athanasiou (2013) examines the assignment problem of a private indivisible good by allowing for monetary transfers, and

\footnotetext{
${ }^{1}$ See, for example, Sprumont (1995), Barbera (2001) and Bossert and Weymark (2006) for comprehensive surveys on strategy-proof rules.
} 
characterizes strategy-proof and anonymous rules. $^{2}$

In a similar vein, Tadenuma and Thomson (1993) consider the problem of allocating a single indivisible good when monetary compensations to losers are possible, and characterize consistent, envy-free, and neutral rules. ${ }^{3}$ Tadenuma and Thomson (1995) examine the problem of fairly allocating an indivisible good when monetary compensations to losers are possible, and show the impossibility of finding an envy-free and strategy-proof rule. Then, Ohseto (1999) characterizes the set of rules that satisfy strategy-proofness, equal compensation, demand monotonicity, and individual rationality. ${ }^{4}$ Ohseto (2000) proves that there is no strategy-proof and Pareto optimal rule when monetary compensations are allowed. Fujinaka and Sakai (2009) consider the assignment problem of an indivisible object with monetary transfers, and examine whether possible manipulations (i.e., absence of strategy-proofness) can have a serious impact on the outcome of agents.

The closest study to the present paper is Pápai (2001), which considers the allocation problem of a single indivisible good to one of several agents when monetary compensation is not allowed. Pápai (2001) shows the impossibility of obtaining a strategy-proof rule that satisfies Pareto optimality, non-dictatorship, and non-bossiness. However, she restricts her study to the strict preferences. We make a similar analysis under the full preference domain where agents are allowed to be indifferent to the object, and generalize her results to the full preference domain. For consistency, we use a similar terminology to Pápai (2001).

A closely related literature is the one that studies the assignment problem of multiple indivisible goods. For instance, Pápai (2000) considers the allocation of heterogenous indivisible objects with monotonic preferences, and characterizes the set of strategy-proof, non-bossy, Pareto optimal, and neutral rules. Pápai (2002) provides the characterization of strategy-proof and non-bossy rules with heterogenous indivisible objects. Ehlers and Klaus (2007) studies the problem of assigning indivisible objects to agents without monetary compensations, and characterizes consistent, strategy-proof, and efficient rules. Kesten and Yazici (2010) study the problem of allocating a set of indivisible goods among a set of agents, and propose a strategy-proof and envy-free rule.

Although it is common to encounter situations in which agents are indifferent, most of the prior literature restricts its analysis to the strict preference domain. Yet, in the last decade, researchers have started to study the assignment problems under the full preference domain. For instance, Bo-

\footnotetext{
${ }^{2}$ Anonymity requires that allocations of agents are independent of agents' names.

${ }^{3}$ Consistency means that if some agents and some objects are removed, the allocation of remaining agents stay the same. Envy-freeness requires that no agent prefers another agent's allocation to his own. Neutrality means that allocations of agents are independent of how objects are labeled.

${ }^{4}$ Equal compensation requires that agents who do not receive the indivisible good receive the same amount of monetary compensation. Demand monotonicity means that the receiver of the indivisible good remains unchanged when he increases his demand, and no other agents increase their demand. Individual rationality requires that all agents end up no worse than their current situations.
} 
gomolnaia et al. (2005) consider the problem of efficiently allocating indivisible objects between agents, and show that all efficient outcomes can be generated using Serially Dictatorial rules. Bogomolnaia et al. (2005) prove that a lottery mechanism is efficient, strategy-proof, anonymous, and neutral. Katta and Sethuraman (2006) examine the assignment problem of indivisible objects in a fair and efficient manner, and prove that strategy-proofness is incompatible with efficiency and envy-freeness under the full preference domain. Larsson and Svensson (2006) generalize strategyproof voting rules to the full preference domain. Yilmaz (2009) characterize individually rational, efficient, and envy-free rules under the full preference domain. Alcalde-Unzu and Molis (2011) generalize Top Trading Cycle mechanism to the full prefernce domain. Athanassoglou and Sethuraman (2011) prove that individual rationality, efficiency, and strategy-proofness are incompatible.

The remainder of the paper is organized as follows. We introduce the model and main properties in Section 2. We provide characterizations of strategy-proof rules in Section 3. We conclude in Section 4. We present all proofs in Appendix.

\section{The Model}

There is a single indivisible object and a set $N=\{1, \ldots, n\}$ of finite agents, where $n \geq 2$. The indivisible object can be assigned to an agent or may not be assigned at all. In particular, if the object is assigned to agent $i \in N$, the outcome of the allocation $x=i$; if the object is not assigned at all, the outcome of the allocation $x=0$. Then, the set of possible outcomes is $N_{0}=\{0,1, \ldots, n\}$. An agent $i \in N$ may or may not receive the object, and hence, the allocation of agent $i$ in the outcome $x$ is $x_{i} \in\{0, i\}$.

The preference of agent $i \in N$ over the object is $R_{i}$, where $R_{i}$ is reflexive for each agent $i$, and the strict component of $R_{i}$ is $P_{i}$. If an agent strictly prefers to receive the object, we denote it by $R_{i}=R_{i}^{+}$; if he is indifferent to the object, we denote it by $R_{i}=R_{i}^{0}$; and if he strictly prefers not to receive the object, we denote it by $R_{i}=R_{i}^{-}$. The set of preference profiles for agent $i \in N$ is $\mathfrak{R}_{i} \in\left\{R_{i}^{+}, R_{i}^{0}, R_{i}^{-}\right\}$, where $\mathfrak{R}=\times_{i \in N} \mathfrak{R}_{\mathrm{i}}$. For notational convenience and ease of distinguishing preference types, we will define a binary relationship (a strict partial order) " $\triangleright$ " such that for $i \in N$ and $R_{i} \in \mathfrak{R}_{i}$, we have $R_{i}^{+} \triangleright R_{i}^{0} \triangleright R_{i}^{-}$. For example, when we say that $R_{i}^{+} \triangleright \tilde{R}_{i}$, we mean that $\tilde{R}_{i} \in\left\{R_{i}^{0}, R_{i}^{-}\right\}$; or when we say that $\tilde{R}_{i} \triangleright R_{i}^{-}$, we mean that $\tilde{R}_{i} \in\left\{R_{i}^{+}, R_{i}^{0}\right\}$. We next explain the special cases that will be used for the remainder of the paper. If all agents prefer to receive the object, we denote it by $R^{+}$, where $R^{+}=\left(R_{1}^{+}, \ldots, R_{n}^{+}\right)$; if all agents are indifferent to the object, we denote it by $R^{0}$, where $R^{0}=\left(R_{1}^{0}, \ldots, R_{n}^{0}\right)$; if all agents prefer not to receive the object, we denote it by $R^{-}$, where $R^{-}=\left(R_{1}^{-}, \ldots, R_{n}^{-}\right)$.

If an agent does not want the object or is indifferent, we use $R_{i}^{\neg}$ to denote his preference, where 
$R_{i}^{\neg} \in\left\{R_{i}^{-}, R_{i}^{0}\right\}$. To refer to $R_{i}=R_{i}^{\neg}$, we use the expression "not prefer to receive the object" whereas to refer to $R_{i}=R_{i}^{-}$, we use the expression "prefer not to receive the object". We denote a preference profile by $R \in \Re$ and a subset of preference profile by $R_{C}=\left(R_{i}\right)_{i \in C}$ for $C \subseteq N$. A rule $f: \mathfrak{R} \longrightarrow N_{0}$ is a function that associates a feasible outcome to each preference profile. The outcome of agent $i$ by rule $f$ under the preference profile $R$ is $f_{i}(R)$ i.e., when $f(R)=x$, $f_{i}(R)=x_{i}$.

Next we define the properties studied in the paper. The first one is a standard strategic robustness property, strategy-proofness, which requires that no agent ever gains by misrepresenting his true preferences. For all $i \in N, \tilde{R}_{i} \in \mathfrak{R}_{i}$ and $R \in \mathfrak{R}$, a rule $f$ is strategy-proof if $f_{i}(R) R_{i} f_{i}\left(\tilde{R}_{i}, R_{-i}\right)$. For some $i \in N, \tilde{R}_{i} \in \mathfrak{R}_{i}$ and $R \in \mathfrak{R}$, a rule $f$ is manipulable and agent $i$ can manipulate it at $R$ if $f_{i}\left(\tilde{R}_{i}, R_{-i}\right) P_{i} f_{i}(R)$. If a rule is not strategy-proof, then it is manipulable. The second property is a standard efficiency property: Pareto optimality. A rule is Pareto optimal if there is no other rule that provides weakly better outcomes for all agents and strictly better outcomes for some agents. Formally, a rule $f$ is Pareto optimal if for all $R \in \mathfrak{R}$ there is no $x \in N_{0}$ such that $x_{i} R_{i} f_{i}(R)$ for all $i \in N$, and $x_{j} P_{j} f_{j}(R)$ for some $j \in N$. The third property is a fairness property which requires that there is no agent who receives the object whenever he prefers to receive, and does not receive it whenever he prefers not to: non-dictatorship. The formal definition of dictatorship is as follows: a rule $f$ is dictatorial if there exists $i \in N$ such that whenever $R_{i}=R_{i}^{+}, f(R)=i$ and whenever $R_{i}=R_{i}^{-}, f_{i}(R)=0$. In this case, agent $i$ is called a dictator with respect to $f$. A rule $f$ is non-dictatorial if there is no dictator agent with respect to $f$. The fourth property, non-bossiness, requires that there is no agent who can change another agent's outcome without changing his own outcome. Formally, a rule $f$ is bossy if there exist $i, j \in N$, $\tilde{R}_{i} \in \mathfrak{R}_{i}$ and $R \in \mathfrak{R}$ such that $f_{i}(R)=f_{i}\left(\tilde{R}_{i}, R_{-i}\right)$ and $f_{j}(R) \neq f_{j}\left(\tilde{R}_{i}, R_{-i}\right)$. In this case, $i$ becomes bossy with $j$ (at $R$ ). If a rule $f$ is not bossy, it is non-bossy. The last property requires that every possible outcome occurs (at least) at one preference profile. The formal definition is that a rule $f$ satisfies citizen sovereignty if for all $x \in N_{0}$, there exists $R \in \mathfrak{R}$ such that $f(R)=x$.

Pápai (2001) shows that strategy-proof and non-bossy rules that satisfy citizen sovereignty are Pareto optimal under the strict preference domain. The following example shows that this result does not hold under the full preference domain.

Example 1 Let $N=\{1,2\}$. Let the rule $f$ be such that $f\left(R_{1}^{-}, R_{2}^{+}\right)=2, f\left(R_{1}^{-}, R_{2}^{0}\right)=2$, $f\left(R_{1}^{-}, R_{2}^{-}\right)=0$ and for all $R \in R \backslash\left\{\left(R_{1}^{-}, R_{2}^{+}\right),\left(R_{1}^{-}, R_{2}^{0}\right),\left(R_{1}^{-}, R_{2}^{-}\right)\right\}, f(R)=1$. Agent 1 obtains the object whenever he prefers to do so (or he is indifferent) and he does not obtain it whenever he does not want it.Then, agent 1 does not need to manipulate. When agent 2 prefers to receive the object, he either gets it or cannot get it because agent 1 does so. Moreover, agent 2 does not 
receive the object whenever he prefers not to. Thus, $f$ is strategy-proof. Whenever agent 1 reports $R_{1}^{0}$ or $R_{1}^{+}$, he receives the object, and hence, he cannot be bossy with agent 2 , and agent 2 cannot be bossy with agent 1 . Then, $f$ is non-bossy. All possible outcomes $(x=0,1,2)$ occur at least at one preference profile, and hence, $f$ satisfies citizen sovereignty. In spite of all these properties, $f$ is not Pareto optimal because the outcome can be improved by letting $f\left(R_{1}^{0}, R_{2}^{+}\right)=2$ instead of $f\left(R_{1}^{0}, R_{2}^{+}\right)=1$.

As Example 1 illustrates strategy-proofness, non-bossiness and citizen sovereignty are not sufficient properties for Pareto optimality to hold. Similarly, the results in Pápai (2001) do not directly follow under the full preference domain. Before we provide necessary and sufficient conditions for Pareto optimality to hold, we define more terms that will be used for the rest of the paper. First, an agent is a discard agent if he strictly prefers not to receive the object but receives it i.e., agent $i \in N$ is a discard agent with respect to $f$ if there exists $R_{-i} \in \mathfrak{R}_{-i}$ such that $f\left(R_{i}^{-}, R_{-i}\right)=i$. Second, a rule is forceful if there is an agent who strictly prefers to receive the object, but the object is awarded to another agent who is indifferent to the object i.e., a rule $f$ is forceful if there exist agents $i, j \in N$ and a preference profile $R \in \mathfrak{R}$ such that $R_{i}=R_{i}^{+}, R_{j}=R_{j}^{0}$ and $f(R)=j$. If a rule $f$ is not forceful, it is non-forceful. Third, a rule $f$ is wasteful if there is an agent who strictly prefers to receive the object, but the object is not assigned at all i.e., a rule $f$ is wasteful if there exists an agent $i \in N$ such that $R_{i}=R_{i}^{+}$and $R \in \Re$, and $f(R)=0$. If a rule $f$ is not wasteful, it is non-wasteful. In the context of this study, a rule $f$ is Pareto optimal if and only if there is no discard agent, and $f$ is non-forceful and non-wasteful.

Example 1 demonstrates that strategy-proofness, non-bossiness, and citizen sovereignty are not sufficient for Pareto optimality. We also need non-forcefulness as shown in the following lemma.

Lemma 1 If a rule is strategy-proof, non-bossy, citizen sovereign, and non-forceful, then it is Pareto optimal.

Before characterizing strategy-proof rules, we present lemmas that will be frequently used for the rest of the paper.

Lemma 2 A rule is Pareto optimal if and only if it is non-forceful, non-wasteful, and contains no discard agents.

The following proposition shows that Pareto optimality implies strategy-proofness.

Proposition 1 If a rule $f$ is Pareto optimal, then $f$ is strategy-proof. ${ }^{5}$

\footnotetext{
${ }^{5}$ Klaus (2001) proves a similar result where free disposal is not allowed.
} 


\section{Characterizations of Strategy-proof Rules}

In this section, we characterize strategy-proof rules that satisfy two of the three additional criteria we search: Pareto optimality, non-dictatorship, and non-bossiness. We start by defining the new terms that will be used for these characterizations. The first one is agent-preference pair. An agent-preference pair is a pair in which agent $i$ has preference $R_{i}$ over receiving the object, i.e., for $i \in N$ and $R_{i} \in \mathfrak{R}_{i}, \Pi=N \times \mathfrak{R}_{i}$ is the set of possible agent-preference pairs, and $\left(i, R_{i}\right)$ is an element of this set. If $\{0\}$ refers to the case where the object is not awarded at all, $\Pi_{0}=\Pi \cup\{0\}$. The second term we define is complete hierarchy. A rule $f$ has a complete hierarchy if there exists an injective ranking function $r: \Pi_{0} \rightarrow \mathbb{N}$ such that the following property holds: for all $j \in N \backslash\{i\}$ and $R \in \mathfrak{R}$, if $r\left(i, R_{i}\right)>r\left(j, R_{j}\right)$ and $r\left(i, R_{i}\right)>r(0)$, then $f(R)=i$; and for all $k \in N$, if $r(0)>r\left(k, R_{k}\right)$, then $f(R)=0$. We next define Hierarchical Choice Function.

Definition 1 Let $R, \tilde{R} \in \Re$. A rule $f$ is a Hierarchical Choice Function (HCF) if it has a complete hierarchy with a ranking function $r$ that satisfies the following two conditions:

(a) For all $i \in N$, if $R_{i} \triangleright \tilde{R}_{i}$ and $r\left(i, R_{i}\right)>r(0)$, there exists no $j \in N \backslash\{i\}$ such that $r\left(i, \tilde{R}_{i}\right)>r\left(j, R_{j}\right)>r\left(i, R_{i}\right)$.

(b) For all $i \in N$, if $r\left(i, \tilde{R}_{i}\right)>r(0)>r\left(i, R_{i}\right)$, then $\tilde{R}_{i} \triangleright R_{i}$.

We characterize the strategy-proof and non-bossy rules in the following proposition.

Proposition 2 A rule $f$ is strategy-proof and non-bossy if and only if $f$ is a HCF.

This result is important because it not only characterizes the strategy-proof and non-bossy rules but also helps characterize the strategy-proof rules that satisfy two of the three additional criteria we seek, i.e., Pareto optimal and non-bossy, and non-dictatorial and non-bossy rules.

\subsection{Pareto Optimal and Non-bossy Rules}

Before characterizing the strategy-proof, Pareto optimal, and non-bossy rules, we define the terms that will be used for the rest of the section. The first term is the top agent. The top agent is the agent who receives the object when all agents prefer to receive it i.e., an agent $i$ is the top agent with respect to $f$ if $f\left(R^{+}\right)=i$. We define the second term, Serial Dictatorship, as follows.

Definition 2 A rule $f$ is a Serial Dictatorship if it is a complete hierarchy with $r: \Pi_{0} \rightarrow \mathbb{N}$ that satisfies

(a) For all $i \in N, r\left(i, R_{i}^{+}\right)>r(0)>r\left(i, R_{i}^{-}\right)$,

(b) For all $i \in N$ and $j \in N \backslash\{i\}, r\left(i, R_{i}^{+}\right)>r\left(j, R_{j}^{0}\right)$.

We characterize the Pareto optimal and non-bossy rules in the following proposition. 
Proposition 3 A rule is Pareto optimal and non-bossy if and only if it is a Serial Dictatorship.

The intuition of Proposition 3 is as follows. A Serial Dictatorship satisfies the requirements of Hierarchical Choice Function (HCF), and hence it is a special case of HCF. Then, by Proposition 2, a Serial Dictatorship is strategy-proof and non-bossy. Moreover, Definition 2(a) means that a Serial Dictatorship is non-wasteful with no discard agent. Definition 2(b) means that a Serial Dictatorship is non-forceful. Then, by Lemma 2, a Serial Dictatorship is Pareto optimal. Thus, a Serial Dictatorship is Pareto optimal and non-bossy. On the other hand, if $f$ is Pareto optimal, then it is non-wasteful, non-forceful, and contains no discard agent by Lemma 2. Being non-wasteful with no discard agent is guaranteed by Definition 2(a), and being non-forceful is guaranteed by Definition 2(b). Furthermore, because $f$ is Pareto optimal, it is strategy-proof as well by Proposition 1. Then, by Proposition 2, strategy-proof and non-bossy $f$ is also a HCF, i.e., $f$ has a complete hierarchy with a ranking function $r$. Hence, all requirements of being a Serial Dictatorship are satisfied. Therefore, a Pareto optimal and non-bossy rule is a Serial Dictatorship.

We next give an example for the strategy-proof, Pareto optimal, and non-bossy rules as follows.

Example 2 Let the rule $f$ be such that $f(R)=\left\{\begin{array}{cc}\min \left\{i \in N \mid R_{i}=R_{i}^{+}\right\} & \text {if } R \neq R^{\urcorner} \\ 0 & \text { if } R=R^{\urcorner}\end{array}\right\}$for all $R \in \Re$. The object is not awarded to indifferent agents, and the agents who strictly prefer not to receive it. Then, $f$ is non-forceful and contains no discard agent. Moreover, if there is an agent who strictly prefers to receive the object, the object does not remain unassigned, so $f$ is nonwasteful. Thus, $f$ is Pareto optimal by Lemma 2. When one agent changes his preference, either his own outcome changes or his outcome stays the same, but the other agents' outcomes also stay the same. Hence, $f$ is non-bossy. The first agent obtains the object whenever he prefers to do so, and he does not obtain it whenever he does not want it, and hence, the first agent is a dictator. Thus, $f$ is dictatorial.

A corollary to Proposition 3 along with Lemma 1 is given below:

Corollary 1 A rule is strategy-proof, non-bossy, citizen sovereign, and non-forceful if and only if it is a Serial Dictatorship.

\subsection{Non-dictatorial and Non-bossy Rules}

We first define Constrained Hierarchical Choice Function as follows.

Definition 3 A Constrained $\mathbf{H C F}$ is a Hierarchical Choice Function with ranking function $r$ : $\Pi_{0} \rightarrow \mathbb{N}$ that satisfies one of the two conditions given below: 
(a) There is no top agent;

(b) There is top agent $i \in N$, but there exists $R \in \mathfrak{R}$ such that $r\left(i, R_{i}^{-}\right)>r(0)$ and $r\left(i, R_{i}^{-}\right)>$ $r\left(j, R_{j}\right)$ for all $j \in N \backslash\{i\}$.

We characterize strategy-proof, non-dictatorial, and non-bossy rules in the following theorem.

Proposition 4 A rule $f$ is strategy-proof, non-dictatorial, and non-bossy if and only if it is a Constrained HCF.

The intuition behind Proposition 4 is as follows. A Constrained HCF is a special case of HCF, so it is strategy-proof and non-bossy by Proposition 2. If the first condition of Constrained HCF holds, there is no top agent, so there is no agent who can obtain the object whenever he prefers to do so. If the second condition holds, there is a top agent but he is also a discard agent, so he obtains the object even if he prefers not to do so. Thus, a Constrained HCF is non-dictatorial. On the other hand, a strategy-proof and non-bossy rule is a HCF with a ranking function $r$ by Proposition 2. Moreover, if a rule is non-dictatorial, then there is no agent who receives the object whenever he prefers to do and does not receive it whenever he prefers not to. The former means that there is no top agent because otherwise due to strategy-proof and non-bossy structure of $f$, the top agent should always keep the object as long as he prefers to do so (see the proof of Proposition 4). Thus the former case corresponds to Definition 3(a). The latter means that there is a top agent but he is also a discard agent, which corresponds to Definition 3(b). Therefore, a strategy-proof, non-dictatorial, and non-bossy rule is a Constrained HCF.

We next demonstrate the non-emptiness of the set of strategy-proof, non-dictatorial, and nonbossy rules in the following example.

Example 3 Let rule $f$ be such that $f(R)=0$ for all $R \in \mathfrak{R}$ and agent $i \in N$ be such that

$R_{i}=R_{i}^{+}$. The object is not assigned regardless of the agents' preferences, so no agent can manipulate. Hence, $f$ is strategy-proof. The object is not awarded at any profile, and hence, there is no agent who obtains the object whenever he prefers to do so. Thus, $f$ is non-dictatorial. Since the object is not awarded at all, there is no agent who can change the outcome of another agent, and hence, $f$ is non-bossy. Because $f$ does not assign the object at all even though agent $i$ strictly prefers to receive it, $f$ is wasteful, so $f$ is not Pareto optimal by Lemma 2.

\subsection{Pareto Optimal and Non-dictatorial Rules}

In this section we characterize strategy-proof, Pareto optimal, and non-dictatorial rules. For this characterization, we define a new term, constrained bossy, as follows. A rule is constrained bossy if there exist two agents $i, j \in N$ and preference profiles $R, \widetilde{R} \in \mathfrak{R}$ such that $R_{j}=R_{j}^{+}, f(R)=j$ 
and $f_{i}\left(\widetilde{R}_{i}, R_{-i}\right)=f_{j}\left(\widetilde{R}_{i}, R_{-i}\right)=0$. In this case, agent $i$ is constrained bossy with agent $j$. Recall that if $f\left(R^{+}\right)=i$, agent $i$ is called the top agent with respect to $f$. If there is an agent who is constrained bossy with the top agent, this rule is called a top-bossy rule.

Definition 4 If $f$ is a top-bossy rule in which there is no discard agent, and $f$ is non-forceful and non-wasteful, then $f$ is a Top-Bossy Choice Function.

We characterize the Pareto optimal and non-dictatorial rules as follows.

Proposition 5 A rule $f$ is Pareto optimal and non-dictatorial if and only if it is a Top-Bossy Choice Function.

The intuition behind Proposition 5 is as follows. If $f$ is a Top-Bossy Choice Function, by definition, $f$ is non-forceful and non-wasteful, and requires that there is no discard agent. Then, $f$ is Pareto optimal by Lemma 2. A Pareto optimal rule is also strategy-proof by Proposition 1, so Top-Bossy Choice Function is strategy-proof. In a Top-Bossy Choice Function, the only candidate to be a dictator is the top agent because he is the one who receives the object when all agents prefer to receive it. However, there is another agent who can change the top agent's outcome by changing his own preference i.e., the constrained bossy agent. Then, the top agent does not obtain the object whenever he prefers to obtain it, and hence, the top agent is not a dictator. Thus, Top-Bossy Choice Function is non-dictatorial. Since there is an agent who is constrained bossy with the top agent, a Top-Bossy Choice Function is constrained bossy.

We next give an example for strategy-proof, Pareto optimal, and non-dictatorial rules.

Example 4 Let the rule $f$ be such that (if $n \geq 3$ )

$$
f(R)=\left\{\begin{array}{cc}
\min \left\{i \in N \mid R_{i}=R_{i}^{+}\right\} & \text {If } R_{n}=R_{n}^{+} \\
\max \left\{i \in N \mid R_{i}=R_{i}^{+}\right\} & \text {If } R_{n}=R_{n}^{\neg}, R \neq R^{\urcorner} \\
0 & R=R^{\urcorner}
\end{array}\right\} \text {for all } R \in \Re .
$$

The object is not assigned to agents who are indifferent and prefer not to receive it. Then, $f$ is non-forceful, and there is no discard agent. Moreover, the object is not unassigned when there is an agent who prefers to obtain it, so $f$ is non-wasteful. Thus, $f$ is Pareto optimal by Lemma 2. By Proposition 1, $f$ is also strategy-proof. The first agent obtains the object when all agents prefer to obtain it, and the object is not awarded to the first agent when he prefers not to receive it. Then, the first agent is the only candidate for being a dictator. However, when the last agent does not prefer to receive the object, the first agent cannot obtain it even if he wants to. Then, the first agent is not a dictator. Thus, $f$ is non-dictatorial. Since the last agent can change the first agent's outcome by 
changing his own preference, the last agent is constrained bossy with the first agent. Hence, $f$ is constrained bossy.

As a result of Propositions 3, 4, and 5 we obtain the following result.

Proposition 6 There exists no strategy-proof rule that is Pareto optimal, non-dictatorial and nonbossy simultaneously.

Proposition 6 is a direct result of Proposition 3, which shows that a strategy-proof, Pareto optimal and non-bossy rule is dictatorial; Proposition 4, which shows that a strategy-proof, non-dictatorial and non-bossy rule is not Pareto optimal; and Proposition 5, which shows that a strategy-proof, Pareto optimal and non-dictatorial rule is bossy. The impossibility result is tight except for the following case.

Lemma 3 If $n=2$ and a rule $f$ is Pareto optimal, then $f$ is dictatorial.

Lemma 3 shows that when there are only two agents, Pareto optimality directly implies dictatorship. A corollary to Proposition 6 along with Lemma 1 is as follows:

Corollary 2 If a rule is strategy-proof, non-bossy, citizen sovereign, and non-forceful, then it is dictatorial.

\section{Conclusion}

We analyze the allocation problem of a single indivisible object under the full preference domain when monetary transfers are not possible. We require the allocation rules to be strategy-proof and we also seek for Pareto optimality, non-dictatorship, and non-bossiness. We characterize strategyproof rules, and prove that Pareto optimal and non-bossy rules are dictatorial, non-dictatorial and non-bossy rules are not Pareto optimal, and Pareto optimal and non-dictatorial rules are (constrained) bossy. Thus, a strategy-proof rule cannot satisfy Pareto optimality, non-dictatorship, and non-bossiness simultaneously. Although these four properties are incompatible in a deterministic environment, a random rule that assigns the object to agents with equal probability satisfies them all. Hence, an interesting future research avenue is characterizing such random allocation rules.

\section{Appendix}

\section{A Proofs of Section 2}

\section{A.1 Proof of Lemma 1}

Let $f$ be strategy-proof, non-bossy, citizen sovereign, and non-forceful. Suppose that $f$ is not Pareto optimal. Then, one of the following three cases happens: Case 1 (wastefulness): for some 
$i \in N$, there is a profile $R \in \Re$ such that $R_{i}=R_{i}^{+}$and $f(R)=0$; Case 2 (discard agent): there exists $R_{-i} \in \mathfrak{R}_{-i}$ such that $f\left(R_{i}^{-}, R_{-i}\right)=i$; Case 3 (forcefulness): for some $i, j \in N$, there exists a profile $R \in \Re$ such that $R_{j}=R_{j}^{+}, R_{i}=R_{i}^{0}$ and $f(R)=i$.

- Case 1: Let $R_{1}=R_{1}^{+}$, without loss of generality. Since $f$ is citizen sovereign, there exists a profile $\tilde{R} \in \mathfrak{R}$ such that $f(\tilde{R})=1$. Then, consider the sequence of profiles given below:

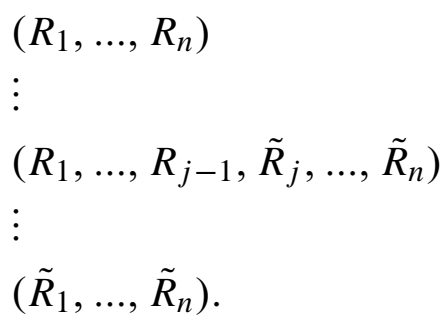

Since $f$ is non-bossy, when $R_{j}$ changes to $\tilde{R}_{j}$, either the outcome does not change or by strategy-proofness, $\tilde{R}_{j} \neq R_{j}^{-}, R_{j} \neq R_{j}^{+}$and $f\left(R_{1}, \ldots, R_{j-1}, \tilde{R}_{j}, \ldots, \tilde{R}_{n}\right)=j$ for $j=$ $1, \ldots, n$. First, $\tilde{R}_{j} \neq R_{j}^{-}$because otherwise, $j$ can manipulate by reporting $R_{j}$ when his actual preference is $\tilde{R}_{j}$. Second, $R_{j} \neq R_{j}^{+}$because otherwise, $j$ can manipulate by reporting $\tilde{R}_{j}$ when his actual preference is $R_{j}$. In the last step, when $R_{1}$ changes to $\tilde{R}_{1}$, we know that $f\left(\tilde{R}_{1}, \ldots, \tilde{R}_{n}\right)=f(\tilde{R})=1$. Then, if $R_{1}=R_{1}^{+}$, agent 1 can manipulate by reporting $\tilde{R}_{1}$ when his actual preference is $R_{1}$. Therefore, $R_{1} \neq R_{1}^{+}$, and this is a contradiction.

Case 2: Let agent 1 be a discard agent without loss of generality i.e., there exists $\tilde{R} \in \mathfrak{R}$ such that $f(\tilde{R})=1$ and $\tilde{R}_{1}=R_{1}^{-}$. Since $f$ is citizen sovereign, there exists $R \in \mathfrak{R}$ such that $f(R)=0$. Then, by the argument in Case 1 , we can establish that $\tilde{R}_{j} \neq R_{j}^{-}$. Using the same reasoning, when $R_{1}$ changes to $\tilde{R}_{1}$ in the last step, $\tilde{R}_{1} \neq R_{1}^{-}$, which is a contradiction.

Case 3: Directly follows from non-forcefulness.

\section{A.2 Proof of Lemma 2}

(a) A Pareto optimal rule $f$ is non-forceful, non-wasteful, and contains no discard agents. Suppose to the contrary that $f$ is either forceful (i) or wasteful (ii) or contains a discard agent (iii). Case (i): $f$ is forceful, then there exist agents $i, j, k \in N$ and a preference profile $R \in \mathfrak{R}$ such that $R_{i}=R_{i}^{+}, R_{j}=R_{j}^{0}$ and $f(R)=j$. Then, the outcome of the allocation $x=i$ is such that $x_{i} P_{i} f_{i}(R), x_{j} R_{j} f_{j}(R)$, and $x_{k} R_{k} f_{k}(R)$ for all $k \in N \backslash\{i, j\}$. Case (ii): $f$ is wasteful, then there exists an agent $i \in N$ and a profile $R \in \Re$ such that $R_{i}=R_{i}^{+}$and $f(R)=0$. Again the outcome of the allocation $x=i$ is such that $x_{i} P_{i} f_{i}(R)$ and $x_{j} R_{j} f_{j}(R)$ for all $j \in N \backslash\{i\}$. Case (iii): $f$ contains a discard agent, then there exists $i \in N, R_{i} \in \mathfrak{R}_{i}$, and $R_{-i} \in \mathfrak{R}_{-i}$ such that $f\left(R_{i}^{-}, R_{-i}\right)=i$. Then, $x_{i}=0$ is such that $x_{i} P_{i} f_{i}(R)$ and $x_{j} R_{j} f_{j}(R)$ for all $j \in N \backslash\{i\}$. 
(b) If a rule $f$ is non-forceful, non-wasteful, and contains no discard agents, then $f$ is Pareto optimal. Suppose to the contrary that $f$ is not Pareto optimal. Then, there exists $x \in N_{0}$ and $R \in \Re$ such that $x_{i} R_{i} f_{i}(R)$ for all $i \in N$, and $x_{j} P_{j} f_{j}(R)$ for (at least) an agent $j \in N \backslash\{i\}$. There are three cases: $R_{j}=R_{j}^{0}, R_{j}=R_{j}^{-}$, and $R_{j}=R_{j}^{+}$. Case (i): $R_{j}=R_{j}^{0}$, agent $j$ cannot be strictly better off under any circumstances. Case (ii): $R_{j}=R_{j}^{-}$and $f(R)=j$, then $x_{j}=0$ is such that $x_{j} P_{j} f_{j}(R)$ and $x_{i} R_{i} f_{i}(R)$ for all $i \in N \backslash\{j\}$. However, $R_{j}=R_{j}^{-}$and $f(R)=j$ means that $j$ is a discard agent, which is a contradiction. Case (iii): $R_{j}=R_{j}^{+}$and $f_{j}(R)=0$. Then $x=j$ is such that $x_{j} P_{j} f_{j}(R)$ and $x_{i} R_{i} f_{i}(R)$ for all $i \in N \backslash\{j\}$ if a) $f(R)=0$ or b) $f(R)=i$ where $R_{i}=R_{i}^{0}$ or c) $f(R)=i$ where $R_{i}=R_{i}^{-}$. Case a) $R_{j}=R_{j}^{+}$and $f(R)=0$ contradicts with non-wastefulness of $f$. Case b) $R_{j}=R_{j}^{+}$and $f(R)=i$ where $R_{i}=R_{i}^{0}$ contradicts with non-forcefulness of $f$. Case c) $f(R)=i$ where $R_{i}=R_{i}^{-}$means that $i$ is a discard agent, which is a contradiction.

\section{A.3 Proof of Proposition 1}

Let $f$ be a Pareto optimal rule. Suppose to the contrary that $f$ is not strategy-proof. Then, there exists an agent $i \in N, \tilde{R}_{i} \in \mathfrak{R}_{i}$ and $R \in \mathfrak{R}$ such that $f_{i}\left(\tilde{R}_{i}, R_{-i}\right) P_{i} f_{i}(R)$. There are three cases: $R_{i}=R_{i}^{0}, R_{i}=R_{i}^{-}$, and $R_{i}=R_{i}^{+}$. If $R_{i}=R_{i}^{0}$, agent $i$ cannot manipulate $f$ because he cannot be strictly better off under any circumstances. If $R_{i}=R_{i}^{-}, f(R)=i$, and $f_{i}\left(\tilde{R}_{i}, R_{-i}\right)=0$, then $f_{i}\left(\tilde{R}_{i}, R_{-i}\right) P_{i} f_{i}(R)$, so $i$ can manipulate $f$ by reporting $\tilde{R}_{i}$. However, $R_{i}=R_{i}^{-}, f(R)=i$ means that $i$ is a discard agent, and by Lemma 2, this contradicts with Pareto optimality of $f$. If $R_{i}=R_{i}^{+}$, $f_{i}(R)=0$, and $f\left(\tilde{R}_{i}, R_{-i}\right)=i$, then $f_{i}\left(\tilde{R}_{i}, R_{-i}\right) P_{i} f_{i}(R)$, so $i$ can manipulate $f$ by reporting $\tilde{R}_{i}$. In this case, either $\tilde{R}_{i}=R_{i}^{-}$or $\tilde{R}_{i}=R_{i}^{0}$. If $\tilde{R}_{i}=R_{i}^{-}, i$ is a discard agent, which contradicts with Pareto optimality of $f$ by Lemma 2. If $\tilde{R}_{i}=R_{i}^{0}, f$ is forceful, which again contradicts with Pareto optimality of $f$ by Lemma 2 .

\section{B Proofs of Section 3}

Before presenting the proof of Proposition 2, we need to prove interim results Lemma A1, A2, and A3 that will be used in the proof of Proposition 2.

Lemma A1 Let $f$ be a non-bossy rule, $i, j \in N$ and $R \in \mathfrak{R}$. If $f(R)=i$, then for all $\tilde{R} \in \mathfrak{R}$, $f_{j}\left(R_{\{i, j\}}, \tilde{R}_{-\{i, j\}}\right)=0$.

Proof. As agents $k \in N \backslash\{i, j\}$ change preferences from $R$ to $\tilde{R}$, a direct consequence of $f$ 's non-bossiness is that the object is either awarded to agent $k$ or stays at an agent different than $j$.

Before presenting Lemma A2, we need to define a new term as follows. If there exists $R \in \mathfrak{R}$ such that $f(R)=i$, then an agent-preference pair $\left(i, R_{i}\right)$ is more prioritized than another agent- 
preference pair $\left(j, R_{j}\right)$, where $j \in N \backslash\{i\}$. We denote the binary relation being more prioritized by " $\succ$ ", and hence, $\left(i, R_{i}\right) \succ\left(j, R_{j}\right)$.

Lemma A2 Let $f$ be a strategy-proof and non-bossy rule, $i, j \in N, R_{i} \in \mathfrak{R}_{i}$ and $R_{j} \in \mathfrak{R}_{j}$. Suppose that $\left(i, R_{i}\right) \succ\left(j, \tilde{R}_{j}\right)$. Then, for all $\tilde{R}_{i} \in \mathfrak{R}_{i}$ such that $\tilde{R}_{i} \triangleright R_{i}$ (if any), $\left(i, \tilde{R}_{i}\right) \succ\left(j, \tilde{R}_{j}\right)$. Moreover, for all $\tilde{R}_{j} \in \mathfrak{R}_{j}$ such that $R_{j} \triangleright \tilde{R}_{j}$ (if any), $\left(i, R_{i}\right) \succ\left(j, \tilde{R}_{j}\right)$.

\section{Proof.}

Claim 1: $f\left(\tilde{R}_{i}, R_{-i}\right)=i$. Proof. Let $i, j \in N, R_{i} \in \mathfrak{R}_{i}$ and $R_{j} \in \mathfrak{R}_{j}$. Suppose that $\left(i, R_{i}\right) \succ\left(j, \tilde{R}_{j}\right)$. Then, there exists $R_{-\{i, j\}} \in \mathfrak{R}_{-\{i, j\}}$ such that $f(R)=i$. If $R_{i}=R_{i}^{0}$, then because of $f$ 's strategy-proofness, $f\left(R_{i}^{+}, R_{-i}\right)=i$ because otherwise, agent $i$ can report $R_{i}^{0}$ when his actual preference is $R_{i}^{+}$. If $R_{i}=R_{i}^{-}$, then because of $f$ 's strategy-proofness, $f\left(R_{i}^{+}, R_{-i}\right)=i$ and $f\left(R_{i}^{0}, R_{-i}\right)=i$ because otherwise, agent $i$ can misrepresent his preference as $R_{i}^{+}$and $R_{i}^{0}$ respectively when it is $R_{i}^{-}$, which is a contradiction. Therefore, for every $\tilde{R}_{i} \in \mathfrak{R}_{i}$ such that $\tilde{R}_{i} \triangleright R_{i}, f\left(\tilde{R}_{i}, R_{-i}\right)=i$, which leads to $\left(i, \tilde{R}_{i}\right) \succ\left(j, R_{j}\right)$.

Claim 2: $f\left(\tilde{R}_{j}, R_{-j}\right)=i$. Proof. If $f\left(R_{j}^{0}, R_{-j}\right)=i$, suppose to the contrary that $f_{i}\left(R_{j}^{-}, R_{-j}\right)=0$. Then, non-bossiness of $f$ implies $f\left(R_{j}^{-}, R_{-j}\right)=j$. In this case, agent $j$ can misrepresent his preference as $R_{j}^{0}$ to avoid receiving the object while his actual preference is $R_{j}^{-}$. If $f\left(R_{j}^{+}, R_{-j}\right)=i$, suppose to the contrary that $f_{i}\left(R_{j}^{-}, R_{-j}\right)=0$. Then, non-bossiness of $f$ implies $f\left(R_{j}^{-}, R_{-j}\right)=j$. In this case, agent $j$ can misrepresent his preference as $R_{j}^{-}$to receive the object while his actual preference is $R_{j}^{+}$(or he can misrepresent his preference as $R_{j}^{+}$to avoid receiving the object while his actual preference is $R_{j}^{-}$). The same logic prevails if $\tilde{R}_{j}=R_{j}^{0}$ because non-bossiness of $f$ implies $f\left(R_{j}^{0}, R_{-j}\right)=\dot{j}$, and agent $j$ can misrepresent his preference as $R_{j}^{0}$ while his actual preference is $R_{j}^{+}$. This contradicts with the strategy-proofness of $f$, and hence, $f\left(\tilde{R}_{j}, R_{-j}\right)=i$ holds.

Lemma A3 Let $f$ be a strategy-proof and non-bossy rule. For $i \in N$ and $R \in \Re$, if $f(R)=0$, then for all $\tilde{R}_{i} \in \mathfrak{R}_{i}$ such that $R_{i} \triangleright \tilde{R}_{i}$ (if any), $f\left(\tilde{R}_{i}, R_{-i}\right)=0$. Moreover, if $f(R)=i$, then for all $\tilde{R}_{i} \in \mathfrak{R}_{i}$ such that $\tilde{R}_{i} \triangleright R_{i}$ (if any), $f\left(\tilde{R}_{i}, R_{-i}\right)=i$.

Proof. Let $f(R)=0$. For some $j \in N$ and $\tilde{R}_{i} \in \mathfrak{R}_{i}$ such that $R_{i} \triangleright \tilde{R}_{i}$, suppose that $f\left(\tilde{R}_{i}, R_{-i}\right)=j$. Non-bossiness of $f$ implies that $j=i$ i.e., $f\left(\tilde{R}_{i}, R_{-i}\right)=i$. If $f\left(R_{i}^{0}, R_{-i}\right)=0$ and $f\left(R_{i}^{-}, R_{-i}\right)=i$, then agent $i$ can manipulate by reporting $R_{i}^{0}$ to avoid receiving the object when his actual preference is $R_{i}^{-}$. If $f\left(R_{i}^{+}, R_{-i}\right)=0$ and $f\left(\tilde{R}_{i}, R_{-i}\right)=i$, where $R_{i} \triangleright \tilde{R}_{i}$, then agent $i$ can manipulate by reporting $R_{i}^{-}\left(\right.$or $R_{i}^{0}$ ) to receive the object while his actual preference is $R_{i}^{+}$. These manipulations contradict with strategy-proofness of $f$, so the first claim holds. For 
some $\tilde{R}_{i} \in \mathfrak{R}_{i}$ such that $\tilde{R}_{i} \triangleright R_{i}$, suppose $f(R)=i$ and $f_{i}\left(\tilde{R}_{i}, R_{-i}\right)=0$. If $R_{i}=R_{i}^{-}$, agent $i$ can manipulate by reporting $R_{i}^{0}$ (or $R_{i}^{+}$) to avoid receiving the object while his actual preference is $R_{i}^{-}$. If $R_{i}=R_{i}^{0}$, agent $i$ can manipulate by reporting $R_{i}^{0}$ to receive the object while his actual preference is $R_{i}^{+}$. These manipulations contradict with strategy-proofness of $f$, so the second claim holds.

\section{B.1 Proof of Proposition 2}

(a) If $f$ is a Hierarchical Choice Function (HCF) for a ranking function $r$, then it is strategyproof and non-bossy. Let $f$ be a HCF for a ranking function $r$. Suppose to the contrary that an agent $i \in N$ can manipulate $f$ under a preference profile $R . R_{i} \neq R_{i}^{0}$ because an indifferent agent cannot improve his outcome, and hence, cannot manipulate $f$. Then, there are two possible manipulations: $R_{i}=R_{i}^{+}$or $R_{i}=R_{i}^{-}$. If $R_{i}=R_{i}^{+}$and $f(R)=i$, since agent $i$ achieves the best outcome, he does not need to manipulate $f$. If $R_{i}=R_{i}^{+}$, $f_{i}(R)=0$ and $r\left(i, R_{i}^{+}\right)>r(0)$, then there is another agent $j \in N$ such that $f(R)=j$. Then, by definition of complete hierarchy, $r\left(j, R_{j}\right)>r\left(i, R_{i}^{+}\right)$. By the first condition of HCF, $r\left(j, R_{j}\right)>r\left(i, R_{i}^{-}\right)$and $r\left(j, R_{j}\right)>r\left(i, R_{i}^{0}\right)$. If $r(0)>r\left(i, R_{i}^{+}\right)$, by the second condition of HCF, $r>r\left(i, R_{i}^{-}\right)$and $r(0)>r\left(i, R_{i}^{0}\right)$. Either case, agent $i$ cannot receive the object by revealing false preference. If $R_{i}=R_{i}^{-}$and $f_{i}(R)=0$, since this is the best outcome for agent $i$, he does not need to manipulate. If $R_{i}=R_{i}^{-}$and $f(R)=i$, for all $j \in N, r\left(i, R_{i}^{-}\right)>r\left(j, R_{j}\right)$. Then, by the first condition of HCF, agent $i$ will retain the object even if he reports his preference as $R_{i}^{0}$ or $R_{i}^{+}$. This contradicts with the fact that agent $i$ can manipulate $f$. Hence, $f$ is strategy-proof.

Now suppose that $f$ is bossy. Then, there exist $i, j \in N$ such that $i$ is bossy with $j$, i.e., there exists $R \in \Re$ such that $f(R)=j, f_{j}\left(\widetilde{R}_{i}, R_{-i}\right)=0$ and $f_{i}\left(\widetilde{R}_{i}, R_{-i}\right)=0 . f(R)=j$ implies that $r\left(j, R_{j}\right)>r(0)$ and for all $k \in N \backslash\{j\}, r\left(j, R_{j}\right)>r\left(k, R_{k}\right)$. If $f_{j}\left(\widetilde{R}_{i}, R_{-i}\right)=0$, then by using $r\left(j, R_{j}\right)>r(0)$ and for all $k \in N \backslash\{i, j\}, r\left(j, R_{j}\right)>r\left(k, R_{k}\right)$, we have $r\left(i, \tilde{R}_{i}\right)>r\left(j, R_{j}\right)$. We have $r\left(i, \tilde{R}_{i}\right)>r\left(j, R_{j}\right)>r(0)$ and for all $k \in N \backslash\{i\}, r\left(i, \tilde{R}_{i}\right)>$ $r\left(j, R_{j}\right)>r\left(k, R_{k}\right)$, and hence, we have $f\left(\widetilde{R}_{i}, R_{-i}\right)=i$. However, $f\left(\widetilde{R}_{i}, R_{-i}\right)=i$ contradicts with bossiness of $f$. Therefore, $f$ is non-bossy.

(b) A strategy-proof and non-bossy rule is a Hierarchical Choice Function (HCF). Let $f$ be a strategy-proof and non-bossy rule. We construct a ranking function $r$ as follows:

- Initialization: Start with $\Pi=N \times \Re_{i}$ and $\Pi_{0}=\Pi \cup\{0\}$. Let

$$
R_{i}^{\max }=\left\{\text { for all } \tilde{R}_{i} \in \mathfrak{R}_{i} \backslash\left\{R_{i}\right\}, R_{i} \in \Re_{\mathrm{i}} \mid R_{i} \triangleright \tilde{R}_{i}\right\} \text { and } R^{\max }=\left(R_{i}^{\max }\right)_{i=1}^{n} .
$$


Initially, $R^{\max }=R^{+}$and $k=0$. Rank the agent-preference pairs as follows:

- Step 1: For all $R \in \mathfrak{R}$, if $f\left(R^{\max }\right)=0, f(R)=0$. In this case, let $r(0)=3 n+1-$ $k$, and rank the remaining agent-preference pairs in $\Pi_{0}$ arbitrarily from 1 to $3 n-k$ and stop. Otherwise, go to Step 2.

- Step 2: For some agent $i \in N$, if $f\left(R^{\max }\right)=i$, let $r\left(i, R_{i}^{\max }\right)=3 n+1-k$ and $k \leftarrow k+1$. Let $\mathfrak{R}_{i} \leftarrow \mathfrak{R}_{i} \backslash\left\{R_{i}\right\}$ and $\Pi_{0} \leftarrow \Pi_{0} \backslash\left\{\left(i, R_{i}^{\max }\right)\right\}$. If $\mathfrak{R}_{i}=\emptyset$, let $r(0)=$ $3 n+1-k$, and rank the remaining agent-preference pairs in $\Pi_{0}$ arbitrarily from 1 to $3 n-k$ and stop. Otherwise, let $\mathfrak{R} \leftarrow \times_{i \in N} \mathfrak{R}_{i}$, recalculate $R^{\max }$ and return to Step 1 .

To show that $f$ is a HCF, we need to show that the generated ranking function $r$ forms a complete hierarchy and the two conditions of HCF are satisfied. To show that $r$ forms a complete hierarchy, let $R \in \mathfrak{R}$, and suppose $r\left(i, R_{i}\right)>r(0)$ and for all $j \in N \backslash\{i\}$, $r\left(i, R_{i}\right)>r\left(j, R_{j}\right)$. Then, we know from the algorithm that there must exist $\tilde{R} \in \mathfrak{R}$ such that $f\left(R_{i}, \tilde{R}_{-i}\right)=i$ (this must occur before the algorithm stops after $r(0)$ ). Moreover, since the algorithm always considers $f\left(R^{\max }\right)$ at Step 2 and for all $j \in N \backslash\{i\}$, we have $r\left(i, R_{i}\right)>r\left(j, R_{j}\right)$. Then, for all $j \in N \backslash\{i\}$, either $\tilde{R}_{j} \triangleright R_{j}$ or $\tilde{R}_{j}=R_{j}\left(\left(j, R_{j}\right) \mathrm{s}\right.$ have lower rank, which means that we encounter them later in the algorithm, and $\left(R_{i}^{\max }\right)$ gets smaller over time). Since $f\left(R_{i}, \tilde{R}_{-i}\right)=i$, for all $j \in N \backslash\{i\},\left(i, R_{i}\right) \succ\left(j, \tilde{R}_{j}\right)$ by Lemma A1 and definition of $\succ$. Then, for all $j \in N \backslash\{i\}$, we have $\left(i, R_{i}\right) \succ\left(j, R_{j}\right)$ by Lemma A2. Thus, by Lemma A1 and non-bossiness of $f, f(R)=i$. Hence, the first condition of complete hierarchy is satisfied.

For the second condition of complete hierarchy, for all $j \in N$, suppose $r(0)>r\left(j, R_{j}\right)$. This immediately implies that the algorithm generating $r$, stops at Step 1 . Then, there exists $\tilde{R} \in \mathfrak{R}$ such that $f(\tilde{R})=0$, and for all $j \in N$, either $\tilde{R}_{j} \triangleright R_{j}$ or $\tilde{R}_{j}=R_{j}$ (since we consider $R^{\max }$ every time and when $f(\tilde{R})=0$ is encountered, $\left(j, R_{j}\right)$ s are not assigned a rank). By changing $\tilde{R}_{j}$ to $R_{j}$ one by one, and each time using Lemma A3, we have $f(R)=0$. Therefore, $r$ is a complete hierarchy.

The ranking function $r$ first ranks $\left(i, R_{i}^{\max }\right)$ before other possible agent-preference pairs of $i \in N$ and the algorithm that generates ranking function $r$ guarantees the second condition of HCF. Moreover, since $\left(i, R_{i}^{\max }\right)$ is ranked before other agent-preference pairs with smaller preferences of $i \in N$, the first condition of HCF is guaranteed. Note that the first condition does not say anything about the agent-preference pairs with rank lower than $r(0)$, so ranking such pairs arbitrarily does not violate it. Thus, $f$ is a HCF with ranking function $r$. 


\section{B.2 Proof of Proposition 3}

A Serial Dictatorship is Pareto optimal and non-bossy. Let $f$ be a Serial Dictatorship. Since Serial Dictatorship is a special case of Hierarchical Choice Function, $f$ is a HCF. Then, by Proposition $2, f$ is strategy-proof and non-bossy. Definition 2(a) guarantees that $f$ is non-wasteful with no discard agent, and Definition 2(b) guarantees that $f$ is non-forceful. Thus, $f$ is Pareto optimal by Lemma 2.

A Pareto optimal and non-bossy rule is a Serial Dictatorship. Let $f$ be a Pareto optimal and non-bossy rule. As Proposition 1 shows, Pareto optimality implies strategy-proofness. Then, $f$ is strategy-proof and non-bossy, and hence, it is a Hierarchical Choice Function by Proposition 2. Since $f$ is a HCF, it has a complete hierarchy with a ranking function $r: \Pi_{0} \rightarrow \mathbb{N}$. Moreover, by Lemma 2, Pareto optimality also implies that there is no discard agent, and $f$ is non-wasteful and non-forceful. Being non-wasteful with no discard agent is ensured by Definition 2(a), and being non-forceful is ensured by Definition 2(b).

\section{B.3 Proof of Proposition 4}

We will first show that a Constrained HCF is strategy-proof, non-dictatorial, and non-bossy. Let $f$ be a Constrained Hierarchical Choice Function. Since $f$ is a special case of Hierarchical Choice Function, it is strategy-proof and non-bossy by Proposition 2. Moreover, $f$ satisfies one of the following requirements of being a Constrained HCF: (i) there is no top agent or (ii) there is a top agent $i$, but there exists $R \in \mathfrak{R}$ such that $r\left(i, R_{i}^{-}\right)>r(0)$ and $r\left(i, R_{i}^{-}\right)>r\left(j, R_{j}\right)$ for all $j \in N \backslash\{i\}$. If the first requirement holds, then there is no agent who receives the object whenever he prefers to do so, because $f\left(R^{+}\right)=0$. If the second requirement holds, there is top agent $i$, who is the only candidate for being a dictator, and he is a discard agent for some preference profile. Because $i$ receives the object even when he does not want to, $i$ cannot be a dictator. Hence $f$ is non-dictatorial.

Second, we will show that a strategy-proof, non-dictatorial, and non-bossy rule is a Constrained HCF. Let $f$ be a strategy-proof, non-dictatorial, and non-bossy rule. By Proposition $2, f$ is a HCF with a ranking function $r: \Pi_{0} \rightarrow \mathbb{N}$. Since $f$ is non-dictatorial, either there is no agent who receives the object whenever he prefers to do so, or such an agent exists but he receives the object even if he does not want to. If the former case occurs, for all $i \in N$, there exists $R \in \mathfrak{R}$ such that $r(0)>r\left(i, R_{i}^{+}\right)$or $r\left(j, R_{j}\right)>r\left(i, R_{i}^{+}\right)$for some $j \in N \backslash\{i\}$. In this case, we will show that there should not be any top agent. Suppose to the contrary that there is a top agent $i$, i.e., $f\left(R^{+}\right)=i$. Then, by the above property, there exists $R \in \Re$ such that either $r(0)>r\left(i, R_{i}^{+}\right)$or $r\left(j, R_{j}\right)>r\left(i, R_{i}^{+}\right)$for some $j \in N \backslash\{i\}$. In any case $f_{i}\left(R_{i}^{+}, R_{-i}\right)=0$. However, this means that there exists an agent $k \in N \backslash\{i\}$, such that when agent $k$ changes his preference from $R_{k}^{+}$ 
to $R_{k}$, agent $i$ loses the object even though he prefers to receive it. If $k$ is the one that receives the object, $f$ is not strategy-proof. If $k$ does not receive the object either, $f$ is bossy. Thus, there is a contradiction. Hence, there should not exist any top-agent. This condition satisfies the first requirement of Constrained HCF. Now suppose that there is an agent who receives the object whenever he prefers to do but he receives the object even if he does not want to. Then, there exists $R \in \mathfrak{R}$ such that $r\left(i, R_{i}^{-}\right)>r(0)$ and $r\left(i, R_{i}^{-}\right)>r\left(j, R_{j}\right)$ for all $j \in N \backslash\{i\}$. This condition satisfies the second requirement of Constrained HCF. Therefore, $f$ is a Constrained HCF.

\section{B.4 Proof of Proposition 5}

A Top-Bossy Choice Function is Pareto optimal and non-dictatorial. Let $f$ be a Top-Bossy Choice Function. By definition, $f$ is non-forceful, non-wasteful, and contains no discard agent. Then $f$ is Pareto optimal by Lemma 2. To prove that $f$ is non-dictatorial, suppose to the contrary that $f$ is dictatorial. Then, there exists a dictator agent $j \in N$ who receives the object whenever he prefers to do so i.e., the top-agent. Since $f$ is top-bossy, there is an agent $i \in N$ such that $i$ is constrained bossy with $j$ i.e., there exist $R \in \mathfrak{R}$ and $\widetilde{R}_{i} \in \mathfrak{R}_{i}$ such that $R_{j}=R_{j}^{+}, f(R)=j$ and $f_{i}\left(\widetilde{R}_{i}, R_{-i}\right)=f_{j}\left(\widetilde{R}_{i}, R_{-i}\right)=0$. Since $R_{j}=R_{j}^{+}$and $f_{j}\left(\widetilde{R}_{i}, R_{-i}\right)=0$, agent $j$ cannot obtain the object although he prefers to do so. Thus, agent $j$ is not a dictator, which is a contradiction.

A Pareto optimal and non-dictatorial rule is a Top-Bossy Choice Function. Let $f$ be Pareto optimal and non-dictatorial. Pareto optimality of $f$ implies that $f$ is non-forceful, non-wasteful, and contains no discard agent by Lemma 2. Then, we only need to show that $f$ is top-bossy. Suppose to the contrary that $f$ is not top-bossy. By Lemma 2, Pareto optimality implies that the object is awarded at $R^{+}$. Thus, there exists a top-agent $i \in N$ such that $f\left(R^{+}\right)=i$. Pareto optimality also implies strategy-proofness of $f$ by Proposition 1. Since $f$ is strategy-proof and not top-bossy, $f\left(R_{i}^{+}, R_{-i}\right)=i$. Moreover, by Lemma 2, Pareto optimality requires that there is no discard agent i.e., $f_{i}\left(R_{i}^{-}, R_{-i}\right)=0$. Then, agent $i$ receives the object whenever he prefers to do so, and does not receive it whenever he prefers not to. Therefore, agent $i$ is a dictator, which is a contradiction.

\section{B.5 Proof of Proposition 6}

It is a direct consequence of Proposition 3 and Propositions 4 and 5.

\section{B.6 Proof of Lemma 3}

Suppose that $n=2$ and a rule $f$ is Pareto optimal. Suppose to the contrary that $f$ is non-dictatorial. By Lemma 2, Pareto optimality implies that there is no discard agent, and $f$ is non-wasteful and non-forceful. Because of non-wastefulness, $f\left(R^{+}\right)>0$, and suppose that $f\left(R^{+}\right)=1$ without loss of generality. Since there is no dictator, there must be a case such that $f\left(R_{1}^{+}, R_{2}^{\neg}\right) \neq 1$. Then, 
either $f\left(R_{1}^{+}, R_{2}^{\neg}\right)=0$ or $f\left(R_{1}^{+}, R_{2}^{\neg}\right)=2$ holds. However, $f\left(R_{1}^{+}, R_{2}^{\neg}\right)=0$ contradicts with nonwastefulness. If $f\left(R_{1}^{+}, R_{2}^{-}\right)=2$, then either $f\left(R_{1}^{+}, R_{2}^{-}\right)=2$ or $f\left(R_{1}^{+}, R_{2}^{0}\right)=2$. If the former one holds, agent 2 is a discard agent, which is a contradiction with Pareto optimality. If the latter one holds, $f$ is forceful, so there is again a contradiction with Pareto optimality. Therefore, when there are two agents, Pareto optimality implies dictatorship.

\section{References}

A. Erdil, H. Ergin, What's the matter with tie-breaking? improving efficiency in school choice, American Economic Review 98 (3) (2008) 669-689.

Y. Sprumont, Strategy-proof collective choice in economic and political environments, The Canadian Journal of Economics 28 (1) (1995) 68-107.

S. Barbera, An introduction to strategy-proof social choice functions, Social Choice and Welfare 18 (2001) 619-653.

W. Bossert, J. Weymark, Social choice: Recent developmentsWorking paper.

J. Glazer, C. A. Ma, Efficient allocation of a prize - king solomon's dilemma, Games and Economic Behavior 1 (1989) 222-233.

M. Perry, P. Reny, A general solution to king solomon's dilemma, Games and Economic Behavior 26 (1999) 279-285.

W. Olszewski, A simple and general solution to king solomon's problem, Games and Economic Behavior 42 (2003) 315-318.

E. Athanasiou, A solomonic solution to the problem of assigning a private indivisible good, Games and Economic Behavior 82 (2013) 369-387.

K. Tadenuma, W. Thomson, The fair allocation of an indivisible good when monetary compensations are possible, Mathematical Social Sciences 25 (1993) 117-132.

K. Tadenuma, W. Thomson, Games of fair division, Games and Economic Behavior 9 (1995) 191-204.

S. Ohseto, Strategy-proof allocation mechanisms for economies with an indivisible good, Social Choice and Welfare 16 (1999) 121-136.

S. Ohseto, Strategy-proof and efficient allocation of an indivisible good on finitely restricted preference domains, International Journal of Game Theory 29 (2000) 365-374.

Y. Fujinaka, T. Sakai, The positive consequences of strategic manipulation in indivisible good allocation, International Journal of Game Theory 38 (2009) 325-348.

S. Pápai, Strategy-proof single unit award rules, Social Choice and Welfare 18 (2001) 785-798.

S. Pápai, Strategy-proof multiple assignments using quotas, Review of Economic Design 5 (2000) 91-105. 
S. Pápai, Strategy-proof and nonbossy multiple assignments, Journal of Public Economic Theory 3 (3) (2002) 257-271.

L. Ehlers, B. Klaus, Consistent house allocation, Economic Theory 30 (2007) 561-574.

O. Kesten, A. Yazici, The pareto-dominant strategy-proof and fair rule for problems with indivisible goods, Economic Theory.

A. Bogomolnaia, R. Deb, L. Ehlers, Strategy-proof assignment on the full preference domain, Journal of Economic Theory 123 (2005) 161-186.

A. Bogomolnaia, H. Moulin, R. Stong, Collective choise under dichotomous preferences, Journal of Economic Theory 122 (2005) 165-184.

A. Katta, J. Sethuraman, A solution to the random assignment problem on the full preference domain, Journal of Economic Theory 131 (2006) 231-250.

B. Larsson, L. G. Svensson, Strategy-proof voting on the full preference domain, Mathematical Social Sciences 52 (2006) 272-287.

O. Yilmaz, Random assignment under weak preferences, Games and Economic Behavior 66 (1) (2009) 546-558.

J. Alcalde-Unzu, E. Molis, Exchange of indivisible goods and indifferences: The top trading absorbing sets mechanisms, Games and Economic Behavior 73 (2011) 1-16.

S. Athanassoglou, J. Sethuraman, House allocation with fractional endowments, International Journal of Game Theory 40 (2011) 481-513.

B. Klaus, Coalitional strategy-proofness in economies with single-dipped preferences and the assignment of an indivisible object, Games and Economic Behavior 34 (2001) 64-82. 